\title{
High carrier injection for all-silicon laser
}

\author{
H. Toufik ${ }^{1}$, W. Tazibt ${ }^{1,2}$, N. Toufik ${ }^{1}$, M. El Tahchi ${ }^{1}$, F. Pélanchon ${ }^{1}$, and P. Mialhe ${ }^{1, a}$ \\ ${ }^{1}$ PROMES-ELIAUS, Laboratoire Euro-Méditerranéen Sciences et Technologies, Université de Perpignan Via Domitia, \\ 52 avenue Paul Alduy, 66860 Perpignan Cedex, France \\ ${ }^{2}$ Department of Physics, University A. Mira, Béjaïa, Algeria
}

Received: 2 November 2011 / Accepted: 12 March 2012

Published online: 23 April 2012 - (C) The Author(s) 2012

\begin{abstract}
This work provides a novel approach for enhanced radiative transitions in silicon microelectronics devices. A process based on hot carrier injection is monitored for the creation of a low dimensions defect layer near the emitter-base interface of bipolar transistors. New energy levels are induced together with a potential barrier. Carrier confinement is correlated with the potential barrier height. The increase of light emission is related to high injection effects in the junction. Results present an advance toward Si-based optoelectronic devices.
\end{abstract}

\section{Introduction}

Silicon is the constituent of more than $95 \%$ of microelectronics systems. It is cheap, abundant in nature and has the necessary mechanical properties to be processed into high-quality electronic devices. The performance of silicon devices results from very low size for large-scale integrated circuits with high reliability.

New applications in telecommunications, audio-visual practices and in the field of game machines drive the market and require further increase of speed for transmission, higher qualities of information and image resolution. A laser light source built from silicon would provide the ideal improvement for these applications for future highspeed electronics. Silicon has an indirect band gap structure [1] which limits its efficiency as a light emitter. At present, laser diodes made with III-V compounds [2] are used to convert signals [3] for the purpose of communication systems with optical fibers, but the incorporation of such compounds in silicon layers leads to distortions of the signals, degradation of sensitivity and limits the reliability.

Since the beginning of this century, silicon has been considered as a material option for photonics. Pavesi et al. [4] realized light amplification with silicon dots implanted into silicon dioxide layer on Si substrates and discussed a model of laser structure with optical pumping between $\mathrm{Si} / \mathrm{SiO}_{2}$ interface states. Many published works have since been considered for silicon optoelectronic applications. The significant advancements can be classified as follows: (I) increasing the silicon radiative recombination rate, (II) developing photonic silicon waveguides or (III) laser cavities' structure. Each is discussed in turn:

(I) Introduction into silicon layers of selected doping material together with realization of quantum confinement

\footnotetext{
${ }^{a}$ e-mail: mialhe@univ-perp.fr
}

has been considered [5-7] in order to increase the radiative recombination rate. Light-emitting molecules or ions have been injected in nano-structures in order to modify the emission properties of the photonic crystal; Liu et al. [8] obtained a high-speed silicon modulator. Reed [9] introduced the idea of "optical circuits" as light emitters, modulators, amplifiers, detectors, to realize photonic devices.

(II) Technology for the development of photonic devices $[10,11]$ led to the emergence of waveguides obtained as a periodic crystalline structure. Photonic crystal structures for integrated optics [12] and applications with silicon-on-insulator technology for waveguide photonic were reviewed in reference [13]. Krauss [14] discussed applications of photonic crystal slow light waveguides. Point defects have been observed [5] to modify the electromagnetic properties of the waveguide introducing photonic microcavities. The selective transmission of heterostructures, consisting of multiple photonic waveguides, in photonic crystals $[15]$ is related to properties of high-quality nanocavities. These works show that the properties of silicon are sensitive to its structure and new photonic devices [5] are expected from exploitation of observed silicon crystal properties at the nanometer scale, such as quantum size effects, light guidance and nonlinear optical properties.

(III) Significant results for application to produce laser action have been published using Raman amplification in silicon [16] with an external laser pump, and confinement within a waveguide has been considered to be implanted within a silicon device. El Tahchi et al. present in [17] an advance in optoelectronic silicon-based applications: a nano-defect structure has been created which boosts the radiative recombination processes and leads to a localized silicon-emitting process, in the infrared region, with the junction polarized for high injection operations. A light amplification is observed when increasing the 
junction forward current. An interesting work [18] from O'Brien et al. reports on photonic crystal microcavities with membrane devices on sapphire for laser applications giving an advance in understanding layers' properties.

These works open the access to a future single-photon technology [19,20].

All results coalesce to point out that localized defects in small structures enhance silicon emission efficiency and that the radiative recombination rate is increased by carriers' confinement. All the interpretations of these results are concerned by quantum effects and strong interactions either between carriers or with the neighborhood. Integration of a photonic circuit into a standard microelectronic process has so far failed to create a silicon laser diode. At the end of the last decade, the challenge is still electrically to stimulate nanocrystals and photonic structures in order to manage/produce a beam of laser light.

This paper presents an advance in optoelectronic silicon devices since it introduces and formulates a process for the creation of an optical active layer inside silicon devices. The emitter-base $n$ - $p$ junction of a microelectronic device is operated in high injection conditions. A degradation of the structure is induced by hot carriers' injection. The model is based on a population inversion associated with a defect layer for carrier confinement and an electrical stimulation of light is demonstrated.

\section{Generation and description of defect layers}

In this work, defect layers have been created by managing the injection of energetic carriers through a silicon $n-p$ junction. Commercial npn transistors were used. Hot carriers were produced by the application of a reverse bias to the emitter-base junction. Both the intensity of the reverse current and the duration of the applied bias were controlled by using a source-measurement unit (Keithley 2400).

Interactions of hot carriers with the silicon layer produce degradation processes near the junction $[21,22]$, which result in a generation of defects near the space charge region. When the emitter-base junction is reversebiased, the electric field in the space charge region is high enough to heat the mobile carriers, charge. These hot carriers can acquire sufficient energy to break weak bonds in the bulk of the junction and at interface layers giving rise to the insertion of defects in the base near the space charge region. The defects disturb the lattice periodicity by creating energy states situated in the band gap of the semiconductor, and engender a defect layer in the base characterized by localized traps and a density of recombination centers. An evolution of the current-voltage $I(V)$ characteristic of the junction is induced and could then be measured for the purpose of monitoring the injection process.

The injection process was monitored by the analysis of the $I(V)$ curve with the classical exponential model $[1,23]$.

The junction parameters' determination permits us to follow the variations of the reverse junction recombination current and of the junction ideality factor $(n)$.

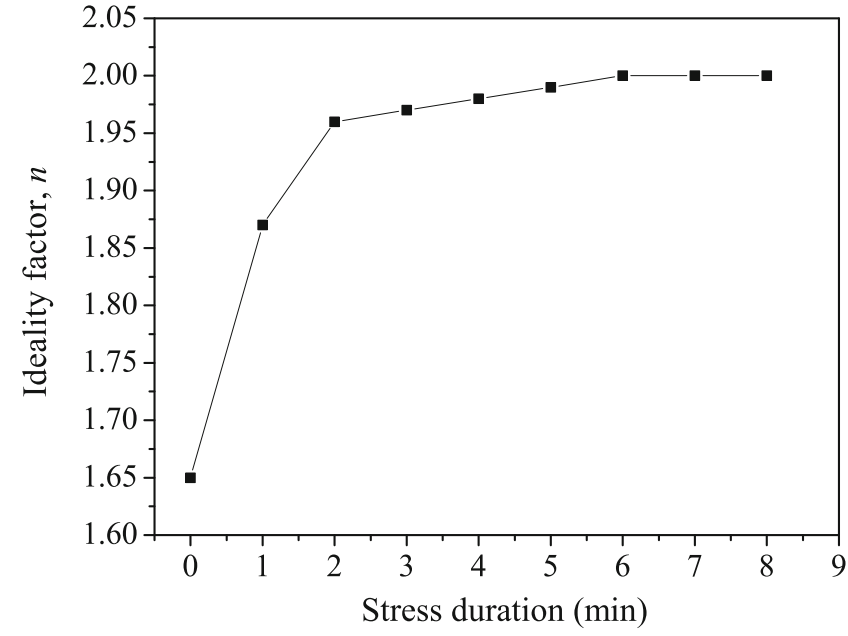

Fig. 1. The junction ideality factor, $n$, vs. duration of the hot carrier injection. The increase of $n$ saturates for the value $n=2$ which indicates high injection effects in the junction.

The increase of $n$ is an indication [22] of recombination process through the generated defects, which induces degradation of the junction properties.

Figure 1 shows the increase of the junction ideality factor as a function of the duration of the hot carrier injection performed with a reversed constant polarization of 7.5 $\mathrm{V}$ applied to the emitter-base junction of a commercial $2 \mathrm{~N} 2222$ transistor. This increase of $n$ means degradation of the junction. This degradation effect saturates since the ideality factor stabilizes at the value $n=2$. It is indicating that high injection effects appear [23] at high voltage operations with minority carrier concentration of the order of majority carrier concentration.

The defect layer assimilated to the degraded region of the $p$ bulk $[24,25]$ can be characterized [25] by a doping level $N_{A}^{d}$ lower than the base doping $N_{A}$, typically of the order of $10^{14} \mathrm{~cm}^{-3}$. The interface with the silicon $p$ type forms a potential barrier which leads, in operating conditions, to confine carriers in the defect layer. When a current flows through the device, the minority carrier density in the bulk is increased. Since the doping level $\left(N_{A}^{d}\right)$ in the defect layer is rather low, this carrier density variation modifies the local potential in the defect region, and the free carriers partially compensate the charge in the defect layer. Under operating conditions, when the applied potential increases, the carrier density increases and a confinement effect appears which compensates the ionization charge inside the defect space-charge region. Then, the potential barriers at the bulk-defect interface decrease and high injection effects are generated.

The interface between the implanted defect and the bulk may be described as a space charge region. Then, using the usual model [1] of an abrupt potential barrier, it is easy to compute the barrier height $\left(V_{D L}\right)$. Values of $V_{D L}$ have been obtained $\left(N_{A}=10^{17} \mathrm{~cm}^{-3}, N_{A}^{d}=10^{14} \mathrm{~cm}^{-3}\right)$, $V_{D L}=179 \mathrm{mV}$ without any external applied potential, $V_{D L}=67 \mathrm{mV}$ with $0.7 \mathrm{~V}$ applied potential and $V_{D L}=0 \mathrm{~V}$ in high injection process. These values may also be 


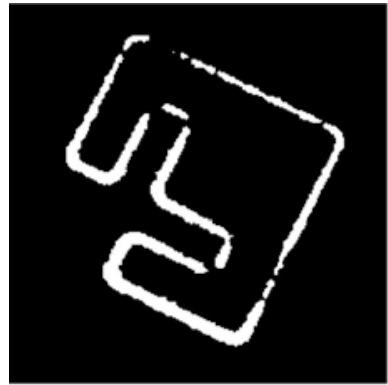

(a)

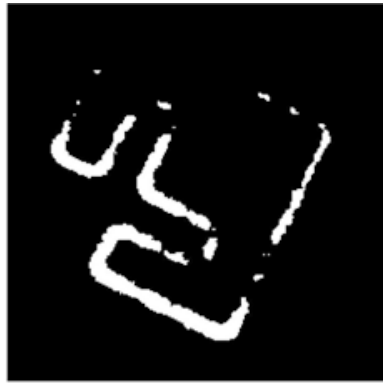

(b)
Fig. 2. Light emission along the base-emitter junction, (a) before and (b) after a $20 \mathrm{~s}$ electrical stress.

computed using PC1D software [26]. They show that in normal operating conditions (applied potential higher than $0.7 \mathrm{~V}$ ), the minority carrier transport is not affected by the defect layer which introduces a carrier confinement in the implanted layer.

\section{Light amplification by electrical stimulation}

For experimental measurements, the silicon device was a commercial bipolar transistor $2 \mathrm{~N} 2222$. An electrical stressing at constant reverse voltage, $7.5 \mathrm{~V}$, was performed on the emitter-base junction. A defect layer was created by injection of hot carriers. The light emission at the device surface was observed during the stress. The currentvoltage characteristics were measured using the Keithley 487 digital picoammeter and a power supply HP 6114A was used to drive the junction into avalanche region. A CCD camera with $500 \times 582$ pixels was used to analyze the emitted light intensity. For imagery, the experimental setup included an optical microscope. A digital thermometer (NiCr-Ni thermocouple) was used to monitor the temperature of the device.

A light emission was observed along the emitter-base junction. The images of the active region taken by the CCD camera have been analyzed and Figure 2 shows the light emission, before stress Figure $2 \mathrm{a}$ and after $20 \mathrm{~s}$ of stress Figure $2 \mathrm{~b}$. The width of the emissive surface increases and the spatial distribution of illuminating surfaces changes during the stress, high intensity appears in some local areas and the light intensity decreases on other parts of the junction surface. This effect has been previously described $[27,28]$. No significant modification of the total light intensity for the whole junction surface was measured.

We have examined the emission of light in the area where high intensity sites appear; the measured light intensity per unit area is displayed in Figure 3 against the duration of the stress experiment.

An increase of the emitted light intensity is measured when increasing the duration of the applied reversed polarization and the emission stabilizes after $90 \mathrm{~s}$ of stress. The light intensity per unit area increases by a factor of 1.7 after $70 \mathrm{~s}$ of stress, compared to the value before stress.

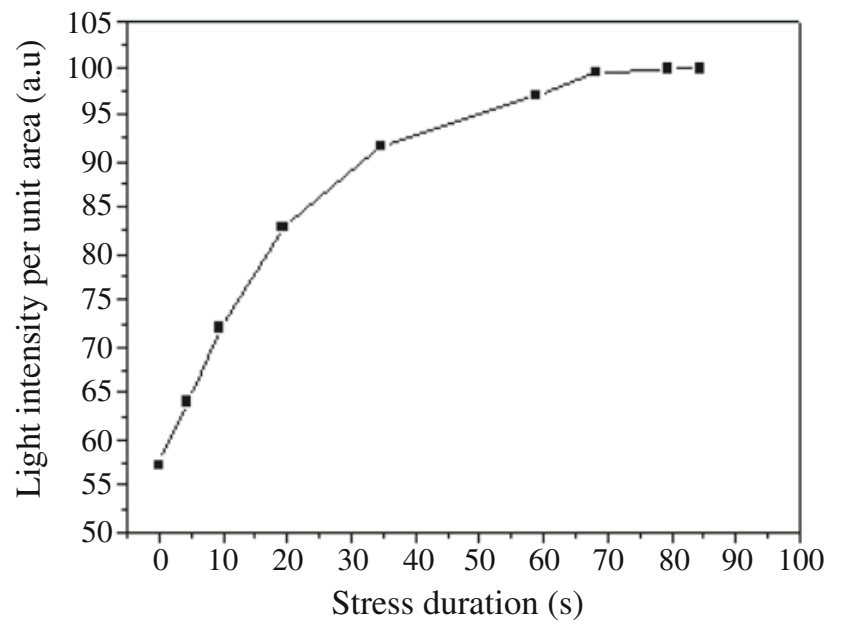

Fig. 3. Light intensity per unit area of the emitter-base junction as a function of the duration of the applied electrical stress (2N2222 bipolar transistor). Measurements were performed on the parts where the width of the emitted surface is increasing along the stress.

The intensity of the reverse current decreases from $80 \mathrm{~mA}$ to $30 \mathrm{~mA}$ after $90 \mathrm{~s}$ of stress.

These results lead us to infer that the emission process is related to the modification of the crystal structure coming from the polarization of the junction into avalanche region. The stabilization of the light emission is correlated with the degradation process since it corresponds to saturation effects of the increase (Fig. 1) of the junction ideality factor to value $n=2$.

Then, the light amplification in the microelectronic silicon devices could be attributed to both local modifications of energy level structure and confinement of carriers. New energy levels for electrons appear in the defect layer, they are related to point defects and confined processes that modify the energy band states compared to classical structures in the bulk silicon crystal. It disturbs minority carrier transport properties and induces [5] new transition probabilities between the bands and individual atomic states.

\section{Conclusion}

This groundwork introduces practical ways for improving the optical properties of silicon devices for optoelectronic applications. Hot carriers' injection has been performed with an applied reverse bias to the emitter-base junction of bipolar transistors in order to create a defect layer in the $p$-type silicon base, located at the emitter-base junction. The process has been controlled by the analysis of the junction $I(V)$ characteristic at each step of the stress. The observed increase of light emission intensity in local area close to the emitter-base junction is related to a carrier confinement, and the defect layer appears as an optical cavity. 
Improvements for integration of optoelectronic applications in silicon microelectronic devices need hard engineering works in the realization of a better optical cavity by controlling the defect generation rate, localization and passivation effects, then stabilization of the layer properties. The energy levels can be calculated using quantum theory for spectral analysis.

These results present an advanced process for implantation of optical functionalities in silicon electronic devices.

\section{References}

1. S.M. Sze, Physics of Semiconductors Devices, 2nd edn. (John Wiley \& Sons, New York, 1981), pp. 13-14

2. M. Mosca, A. Castiglia, H.-J. Bühlmann, J. Dorsaz, E. Feltin, J.-F. Carlin, N. Grandjean, Eur. Phys. J. Appl. Phys. 43, 51 (2008)

3. P. Ball, Nature 409, 974 (2001)

4. L. Pavesi, L.D. Negro, C. Mazzoleni, G. Franzo, F. Priolo, Nature 408, 440 (2000)

5. O. Anopchenko, P. Bettotti, M. Cazzanelli, N. Daldosso, L. Ferraioli, Z. Gaburro, R. Guider, S.M. Hossain, D. Navarro-Urrios, A. Pitanti, S. Prezioso, R. Spano, M. Wang, L. Pavesi, IEEE Int. Semicond. Conf. 1, 175 (2007)

6. J. Bao, M. Tabbal, T. Kim, S. Charnvanichborikarn, J.-S. Williams, M.-J. Aziz, F. Capasso, Optic. Express 15, 6727 (2007)

7. G. Davies, Phys. Rep. 176, 83 (1989)

8. A. Liu, R. Jones, L. Liao, D. Samara-Rubio, D. Rubin, O. Cohen, R. Nicolaescu, M. Paniccia, Letters to Nature 427, 615 (2004)

9. G.T. Reed, Nature 427, 595 (2004)

10. P.K. Bhattacharya, J. Phys. D: Appl. Phys. 40 (2007)

11. Z.-G. Huang, J. Phys. D: Appl. Phys. 44, 245406 (2011)
12. W. Zhou, Z. Qiang, L. Chen, J. Phys. D: Appl. Phys. 40, 2615 (2007)

13. R. Dekker, N. Usechak, M. Forst, A. Driessen, J. Phys. D: Appl. Phys. 40, R249 (2007)

14. T.K. Krauss, J. Phys. D: Appl. Phys. 40, 2666 (2007)

15. D. Lindley, Phys. Rev. E 78, 045603 (2008)

16. J. Faist, Nature 433, 691 (2005)

17. M. El Tahchi, E. Nassar, P. Mialhe, Microelectronics 36, $260(2005)$

18. J. O'Brien, W. Kuang, J.-R. Cao, M.-H. Shih, P.-T. Lee, M. Bagheri, A. Mock, W.K. Marshall, J. Phys. D: Appl. Phys. 40, 2671 (2007)

19. S.A. Castelletto, R.E. Scholten, Eur. Phys. J. Appl. Phys. 41, 181 (2008)

20. M. Orrit, Nature Photonics 4, 667 (2010)

21. N. Toufik, F. Pélanchon, P. Mialhe, J. Electron Devices 1, 7 (2003)

22. W. Tazibt, P. Mialhe, J.P. Charles, M.A. Belkhir, Microel. Reliab. 48, 348 (2008)

23. M. El-Tahchi, N. Toufik, F. Pélanchon, M. Ajaka, A. Khoury, P. Mialhe, J. Phys. D: Appl. Phys. 35, 487 (2002)

24. C.T. Sah, J.Y.C. Sun, U.T. Tzou, J. Appl. Phys. 55, 1525 (1984)

25. G.C. Messenger, M.S. Ash, The Effects of Radiation on Electronic Systems, 2nd edn. (Van Nostrand Reinhold, New York, 1991), pp. 209-222; 805-821

26. P.A. Bassore, IEEE Trans. Electron Devices 37, 337 (1990)

27. M. de la Bardonnie, D. Jiang, S.E. Kerns, D.V. Kerns, P. Mialhe, J.-P. Charles, A. Hoffman, IEEE Trans. Electron Devices 46, 1234 (1999)

28. A. Chatterjee, B. Bhuva, Solid-State Electron. 47, 665 (2003)

Open Access This article is distributed under the terms of the Creative Commons Attribution Noncommercial License which permits any noncommercial use, distribution, and reproduction in any medium, provided the original author(s) and source are credited. 